\title{
Efecto ecotoxicológico del cloruro de potasio sobre la lombriz roja californiana (Eisenia foetida)
}

\author{
Ecotoxicological effect of potassium chloride on the californian red worm \\ (Eisenia foetida)
}

\author{
Alfonso Ramírez Caján ${ }^{1}$, Amelia María Mudarra Pascual ${ }^{2}$, Milagros Jubica Palacín Anco ${ }^{3}$ \\ Recibido: 15/07/2020 - Aprobado: 24/11/2020 - Publicado: 18/06/2021
}

\begin{abstract}
RESUMEN
Esta investigación presenta la metodología para la evaluación de los efectos ecotoxicológicos del cloruro de potasio en la lombriz roja Eisenia foetida, a través de un bioensayo de exposición crónica de 14 días. El material biológico en estadío adulto proveniente de la zona de criadero de lombrices de la Universidad Nacional Agraria La Molina, fue sometido a 5 concentraciones a fin de determinar el $\mathrm{CL}_{50}$ y la mortandad como punto final, evidenciándose deformación, decoloración, inflamaciones y abultamiento anormal en la zona clitelar de las lombrices durante el proceso, llevándose a cabo además la medición de los parámetros ambientales de pH, temperatura y humedad del sustrato (suelo) al 1, 4, 7, 10 y 14 días.

Palabras clave: Bioensayo; clitelio; efectos ecotoxicológicos; Eisenia foetida; mortandad.

ABSTRACT

This research presents the methodology for the evaluation of the ecotoxicological effects of potassium chloride in the red worm Eisenia foetida, through a 14-day chronic exposure bioassay. The biological material in adult stage from the worm hatchery area of the Universidad Nacional Agraria La Molina, was subjected to 5 concentrations to determine the LC50 and mortality as the end point, showing deformation, discoloration, inflammation, and abnormal bulging in the clitellar zone of the worms during the process, also carrying out the measurement of the environmental parameters of $\mathrm{pH}$, temperature, and humidity of the substrate (soil) at 1, 4, 7, 10 and 14 days.
\end{abstract}

Keywords: Bioassay; clithelium; ecotoxicological effects; Eisenia foetida; mortality. 


\section{INTRODUCCIÓN}

Se tiene conocimiento que los bioensayos de toxicidad con lombrices son reconocidos como prueba para suelos contaminados, por lo que en esta investigación se ha considerado a la especie Eisenia foetida, debido a que es una especie de amplia distribución y fácil manejo (Cuevas \& Cerrato, 2002). Las lombrices de tierra contribuyen a mantener la porosidad de los suelos, mejoran la fertilización, y son representativos de los invertebrados que viven en los suelos (Mancebo Rodríguez et al., 2011).

Entonces para conocer previamente una concentración letal de toxicidad de cualquier insumo químico, es necesario realizar un ensayo previo, con un tóxico de referencia, a fin de estimar la sensibilidad de la especie escogida, que en esto caso ha sido la Eisenia foetida, por lo que para la presente investigación se ha visto por conveniente utilizar como tóxico de referencia el cloruro de potasio, debido a su disponibilidad en estado puro, estabilidad en solución, mayor reproducibilidad de los resultados y presentar un menor riesgo de salud, para quien ejecuta la prueba (Gomez Hernandez, 2014).

Conocer la sensibilidad de la Eisenia foetida con el tóxico de referencia cloruro de potasio, permite ver si es apta o no para próximos ensayos de toxicidad de otros compuestos o insumos químicos; asi como determinar la concentración letal media y la mortandad de los organismos, dentro de los parámetros establecidos (Gomez Hernandez, 2014).

\section{MÉTODOS}

\subsection{Diseño del estudio}

Para evaluar la toxicidad del cloruro de potasio sobre Eisenia foetida (tasa de mortalidad), se realizó una investigación experimental, cuyo diseño se basó en un grupo control y un grupo experimental (5 concentraciones), con 10 organismos de prueba por cada unidad muestral. Se emplearon recipientes de plástico transparentes de 11 de capacidad, donde se colocaron $300 \mathrm{~g}$ de suelo húmedo artificial por cada uno, a los cuáles se le añadieron 10 lombrices adultas en buen estado, limpias, secas y pesadas por cada recipiente, cubierto con una malla mosquitera de color negro de $20 \times 20 \mathrm{~cm}$ y asegurado con una liga para evitar el escape de las lombrices. Las lombrices adultas fueron expuestas a 5 concentraciones diferentes de cloruro de potasio $(\mathrm{KCl})$, determinadas mediante la aplicación del factor 0.5 con 4 repeticiones cada una, las mismas que se encontraban mezcladas con el suelo artificial.

Durante la exposición al tóxico en bioensayo subcrónico, el material biológico no recibió alimentación (Chang Marrero O., Jiménez Aguila E., Hernández Castañedo Z., Armengol Hernández R., Meneses-Marcel A., 2018). Se efectuó el registro de datos de la humedad, pH y temperatura del sustrato al 1, 4, 7, 10 y 14 día. En este ensayo se utilizaron un total de 240 lombrices. Se midió la mortandad a través de la concentración letal media $\left(\mathrm{CL}_{50}\right)$, la cual representa la concentración del suelo problema que ocasiona la muerte (daño máximo) en el $50 \%$ de las lombrices que han sido expuestas (Iannacone Oliver, 2017). Se evaluó el $\mathrm{CL}_{50}$ de las lombrices adultas a los 4 , 7, 10 y 14 días del bioensayo, utilizándose como criterio de mortandad el pinchado de las lombrices en el cuerpo con un isopo durante 3 segundos, a fin de verificar algún estímulo o movimiento mecánico del organismo y las características de los individuos sobrevivientes.

\subsection{Instrumentos empleados \\ 2.2.1 Materiales}

Los materiales utilizados fueron un vaso precipitado de 300 y $1000 \mathrm{ml}$, probeta 50 y $100 \mathrm{ml}$, propipeta, pipeta de vidrio de $10 \mathrm{ml}$, termómetro $\left(0-100^{\circ} \mathrm{C}\right)$, embudo mediano, soporte universal con aro, papel filtro Whatman $\mathrm{N}^{\circ} 41$, pisceta, bagueta, placa Petri, pinzas de disección, espátula de acero inoxidable, fiola de $1000 \mathrm{ml}$, taperes transparentes de plástico de 11 (24 unidades), guantes de látex, malla mosquitera de color negro (24 retazos de $20 \times 20 \mathrm{~cm})$ y ligas (24 unidades) (Ver figura 1).

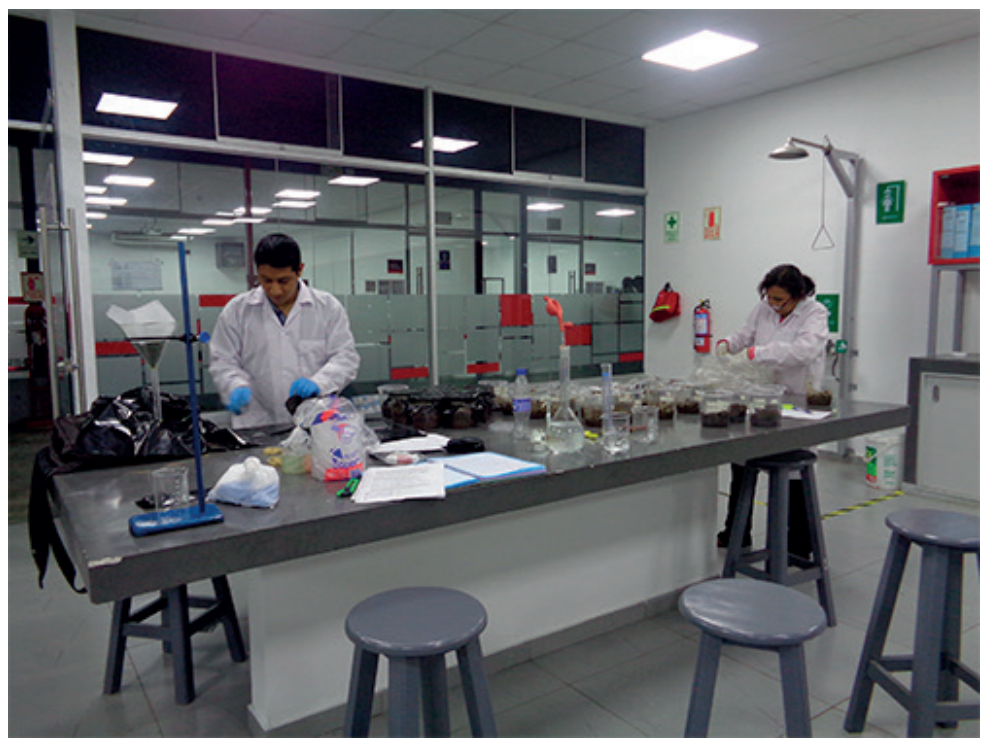

Figura 1. Material de medición y análisis 


\subsubsection{Equipos}

Los equipos utilizados fueron un multiparámetro Hamna (para medir $\mathrm{pH}$ y temperatura), una estufa Daihan (para determinar el contenido de humedad), una balanza analítica Sartorius con capacidad de $220 \mathrm{~g}$, lectura min. de $0.1 \mathrm{mg}$ (para pesar las lombrices y el $\mathrm{KCl}$ ) y una balanza de precisión Sartorius con capacidad de $2100 \mathrm{~g}$, lectura min. de $10 \mathrm{mg}$. (para pesar el suelo húmedo y seco).

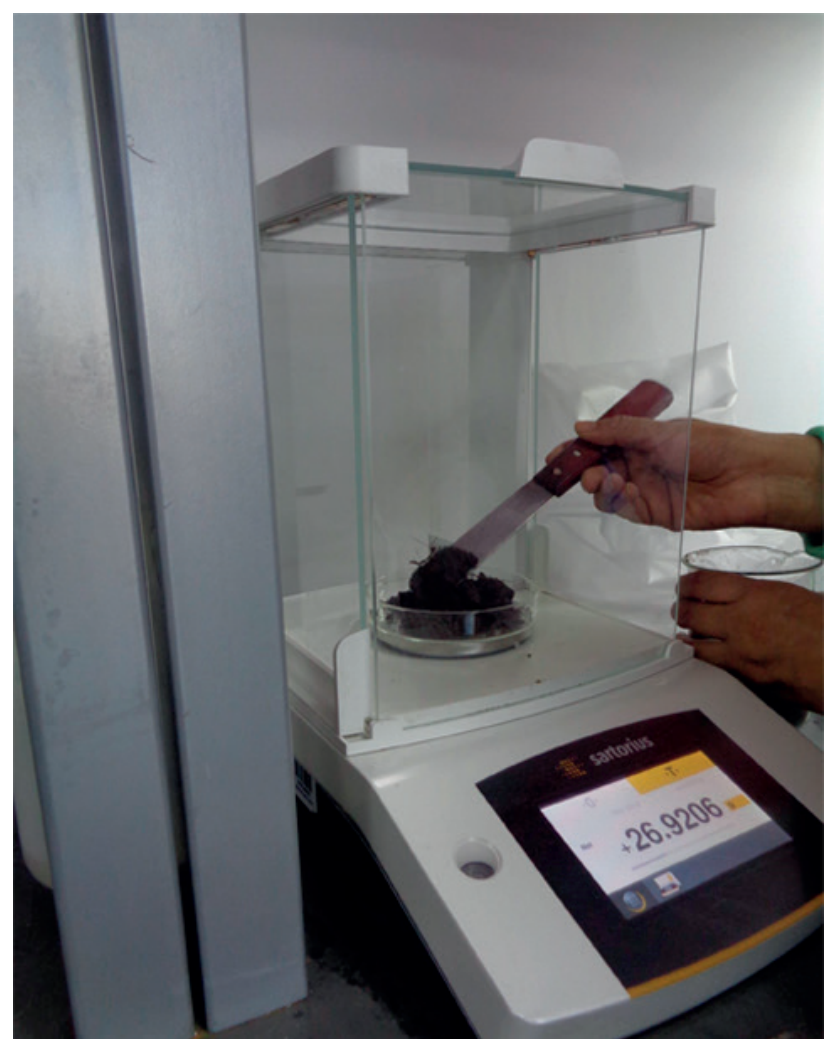

Figura 2. Equipo de medición y lectura

\subsection{Técnicas de colección de datos}

\subsubsection{Organismo de prueba}

Se utilizaron lombrices de tierra de la especie Eisenia foetida provenientes de la Universidad Nacional Agraria La Molina. Se seleccionaron los individuos evitando su manipulación excesiva y disponiéndolas sobre una superficie plana escogiendo las lombrices adultas (cliteladas), en óptimas condiciones físicas (Rubiano Rodríguez, 2019). Las longitudes oscilantes fueron de 7 a $9.8 \mathrm{~cm}$ y con un peso equivalente a $0.5 \pm 0.1 \mathrm{~g}$ (Ver Tablas 1,2 y 3 ). Las lombrices fueron aclimatadas durante 1 día en suelo artificial antes del inicio de los ensayos a una temperatura de $20 \pm 2{ }^{\circ} \mathrm{C}$ y una humedad relativa de $65 \%$ sin suministro de alimento, posteriormente fueron lavadas colocándose en cajas Petri con papel absorbente para que pudieran limpiarse por sí mismas a través de su movimiento (Peña Córdova, 2018). Los bioensayos fueron realizados en el Laboratorio de Biotecnología de la Universidad César Vallejo.

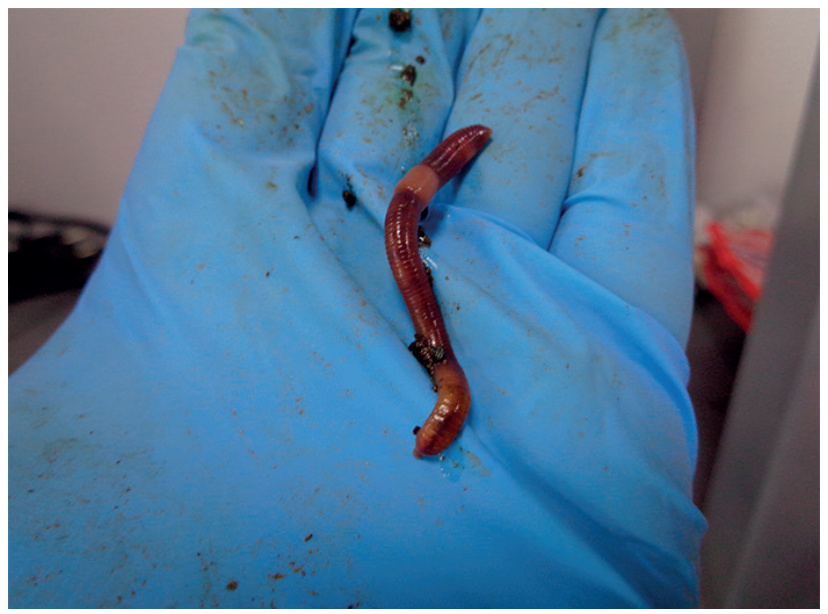

Figura 3. Evaluación de Eisenia foetida

Tabla 1. Aspectos biológicos de la lombriz roja californiana

\begin{tabular}{lll}
\hline \multicolumn{1}{c}{ Aspecto } & \multicolumn{1}{c}{ Unidad } & \multicolumn{1}{c}{ Eisenia foetida } \\
\hline Color & -- & Roja con bandas pardas \\
Tamaño de la especie & $\mathrm{cm}$ & $7.0-9.8$ \\
Peso de la especie & $\mathrm{g}$ & $0.5 \pm 0.1$ \\
Tiempo de madurez & días & 60 \\
\hline Fuente. Elaboración propia &
\end{tabular}

Tabla 2. Medición de longitud promedio de las lombrices rojas

\begin{tabular}{|c|c|c|c|c|c|c|c|c|c|c|}
\hline \multirow{2}{*}{ Recipiente } & \multicolumn{10}{|c|}{ Longitud (cm) } \\
\hline & 1 & 2 & 3 & 4 & 5 & 6 & 7 & 8 & 9 & 10 \\
\hline Control & 7.1 & 8.0 & 8.2 & 9.3 & 8.6 & 9.5 & 8.7 & 8.9 & 7.9 & 9.1 \\
\hline 1 & 7.0 & 7.4 & 7.2 & 9.1 & 8.5 & 8.8 & 9.2 & 8.3 & 8.9 & 8.2 \\
\hline 2 & 7.0 & 8.0 & 8.0 & 9.0 & 8.9 & 9.1 & 8.7 & 8.8 & 8.6 & 8.7 \\
\hline 3 & 9.2 & 7.9 & 7.2 & 8.0 & 7.3 & 9.8 & 9.5 & 9.3 & 9.4 & 9.7 \\
\hline 4 & 8.4 & 8.9 & 8.8 & 9.0 & 9.1 & 9.4 & 9.7 & 9.5 & 9.6 & 9.4 \\
\hline 5 & 9.5 & 7.9 & 7.6 & 7.8 & 8.6 & 8.9 & 9.4 & 9.1 & 9.3 & 8.8 \\
\hline
\end{tabular}

Fuente. Elaboración propia 
Tabla 3. Medición del peso promedio de las lombrices rojas

\begin{tabular}{cccccccccccc}
\hline \multirow{2}{*}{ Recipiente } & \multicolumn{10}{c}{ Peso (g) } \\
\cline { 2 - 27 } & $\mathbf{1}$ & $\mathbf{2}$ & $\mathbf{3}$ & $\mathbf{4}$ & $\mathbf{5}$ & $\mathbf{6}$ & $\mathbf{7}$ & $\mathbf{8}$ & $\mathbf{9}$ & $\mathbf{1 0}$ \\
\hline C0 & 0.45 & 0.50 & 0.51 & 0.59 & 0.54 & 0.58 & 0.52 & 0.56 & 0.49 & 0.56 \\
C1 & 0.40 & 0.47 & 0.43 & 0.56 & 0.53 & 0.55 & 0.59 & 0.53 & 0.55 & 0.50 \\
C2 & 0.40 & 0.49 & 0.47 & 0.55 & 0.57 & 0.57 & 0.53 & 0.55 & 0.53 & 0.54 \\
C3 & 0.59 & 0.48 & 0.45 & 0.44 & 0.43 & 0.60 & 0.56 & 0.56 & 0.57 & 0.60 \\
C4 & 0.52 & 0.51 & 0.52 & 0.52 & 0.58 & 0.58 & 0.60 & 0.58 & 0.59 & 0.57 \\
C5 & 0.58 & 0.43 & 0.43 & 0.47 & 0.50 & 0.51 & 0.57 & 0.57 & 0.58 & 0.53 \\
\hline
\end{tabular}

Fuente. Elaboración propia

\subsubsection{Suelo a utilizar}

El suelo artificial estuvo compuesto por $10 \%$ de materia orgánica, 20\% de arcilla y 70\% de arena, utilizándose un total de 7200 g. Para el ensayo se utilizaron $300 \mathrm{~g}$ de suelo artificial con 4 réplicas por cada concentración ( 5 concentraciones más un control), además se determinaron la temperatura, $\mathrm{pH}$ y humedad del suelo, parámetros cuyas mediciones obtenidas fueron de $20 \pm 2{ }^{\circ} \mathrm{C}, 7 \pm 0.5$ y $20 \%$, respectivamente (Ver tabla 4). La muestra inicial de suelo artificial estaba compuesta por $80 \%$ de tierra seca y $20 \%$ de agua.

Tabla 4. Medición de parámetros ambientales de $\mathrm{pH}$, humedad y temperatura del sustrato

\begin{tabular}{cccc}
\hline \multirow{2}{*}{ Día } & \multicolumn{3}{c}{ Parámetros } \\
\cline { 2 - 4 } & $\mathbf{p H}$ & $\mathrm{T}^{\circ} \mathrm{C}$ & $\mathbf{H} \%$ \\
\hline 1 & 6.66 & 20 & 65.4 \\
4 & 7.25 & 20 & 65.3 \\
7 & 7.67 & 20 & 65.1 \\
10 & 7.85 & 20 & 65.1 \\
14 & 7.96 & 20 & 65.0 \\
\hline
\end{tabular}

Fuente. Elaboración propia

Se realizó la prueba de retención o saturación de agua, para ello se pesó $100 \mathrm{~g}$ de suelo secado previamente en una estufa y se colocó en un embudo con papel filtro el cual estuvo sujeto a un soporte universal, posteriormente se adicionó agua al recipiente midiendo el volúmen gastado cuando cae la primera gota. Teniendo el volúmen que satura el $100 \%$ del suelo, podemos calcular el volúmen necesario para humedecer el suelo en un $65 \%$ (Carlosama Camacho \& Cepeda Rojas, 2018). La humedad estimada para el sustrato fue calculada utilizando como referencia el valor de la capacidad de retención de agua en suelo equivalente a $51.81 \%$, cuyo valor fue multiplicado por un $45 \%$ de humedad requerida y $240 \mathrm{~g}$ de suelo artificial; esto es debido a que al inicio del ensayo el sustrato contenía solo un $20 \%$ de humedad del $65 \%$ considerado; por lo cual el valor promedio necesario para $240 \mathrm{~g}$ de suelo seco fue de $55.95 \mathrm{ml}$, obteniendo aproximadamente $300 \mathrm{~g}$ de suelo húmedo. Todos estos valores se obtuvieron empleando el método gravimétrico en el laboratorio.

\subsubsection{Preparación de soluciones}

Los cálculos para la estimación de la cantidad de volúmen de agua y cloruro de potasio de acuerdo, se realizaron tomando en cuenta la densidad del agua $(1 \mathrm{~g} /$ $\mathrm{ml})$, la densidad de la solución de $\mathrm{KCl}(1,0883 \mathrm{~g} / \mathrm{ml}) \mathrm{y}$ la concentración de la solución $(\mathrm{KCl})$ de $132.65 \mathrm{~g} / \mathrm{L}$ (Ver tabla 5). De esta solución se tomaron los volúmenes que se necesitaban según los cálculos realizados para obtener una muestra preparada de cloruro de potasio de $132.65 \mathrm{~g} / 1$ ( $\mathrm{KCl}$ de grado ACS de marca RJ Baker y agua destilada) y las 5 concentraciones diferentes para $240 \mathrm{~g}$ de suelo seco artificial (Ver figura 4).

a. Determinación de los volúmenes de agua y $\mathrm{KCl}$
para una concentración de $1.5 \mathrm{mg} \mathrm{KCl} / \mathrm{g}$ suelo

Peso KCl: $(240$ g x $1.5 \mathrm{mg} / \mathrm{g}$ x $1 \mathrm{~g}) / 1000 \mathrm{mg}=0.36 \mathrm{mg}$

Vol. KCl: 0.36 g / 132.65 g/L x 1000 ml/L=2.71 ml

$2.71 \mathrm{ml} \times 1,0883 \mathrm{~g} / \mathrm{ml}=2.95 \mathrm{~g}$

Vol. $\mathbf{H}_{2} \mathbf{O}$ : $(55.95 \mathrm{~g}-2.95 \mathrm{~g})$ x $1 \mathrm{~g} / \mathrm{ml}=\mathbf{5 3} \mathbf{~ m l}$

b. Determinación de los volúmenes de agua y $\mathrm{KCl}$ para una concentración de $3 \mathrm{mg} \mathrm{KCl} / \mathrm{g}$ suelo

Peso KCl: $(240$ g x 3 mg/g x 1 g) / 1000 mg = 0.72 mg

Vol. KCl: 0.72 g / 132.65 g/L x 1000 ml/L= $5.43 ~ \mathbf{~ m ~}$

$5.43 \mathrm{ml} \mathrm{x} 1,0883 \mathrm{~g} / \mathrm{ml}=5.9 \mathrm{~g}$

Vol. $\mathbf{H}_{2}$ O: $(55.95 \mathrm{~g}-5.9 \mathrm{~g}) \times 1 \mathrm{~g} / \mathrm{ml}=\mathbf{5 0 . 0 5} \mathbf{~ m l}$

c. Determinación de los volúmenes de agua y $\mathrm{KCl}$ para una concentración de $6 \mathrm{mg} \mathrm{KCl} / \mathrm{g}$ suelo

Peso KCl: $(240$ g x 6 mg/g x 1 g) $/ 1000$ mg = 1.44 mg

Vol. KCl: 1.44 g / 132.65 g/L x 1000 ml/L= 10.86 ml

$10.86 \mathrm{ml} \mathrm{x} 1,0883 \mathrm{~g} / \mathrm{ml}=11.82 \mathrm{~g}$

Vol. H$_{2}$ O: $(55.95 \mathrm{~g}-11.82 \mathrm{~g})$ x $1 \mathrm{~g} / \mathrm{ml}=\mathbf{4 4 . 1 3} \mathbf{~ m l}$

d. Determinación de los volúmenes de agua y $\mathrm{KCl}$ para una concentración de $12 \mathrm{mg} \mathrm{KCl} / \mathrm{g}$ suelo

Peso KCl: (240 g x 12 mg/g x 1 g) /1 000 mg = 2.88 mg 
Tabla 5. Preparación de soluciones

\begin{tabular}{cccccccc}
\hline Concentración & $\begin{array}{c}\mathrm{KCl} \\
\text { (mg KCl/g suelo) }\end{array}$ & Vol. $\mathrm{H}_{2} \mathrm{O}(\mathrm{ml})$ & Vol. $\mathrm{KCl}(\mathrm{ml})$ & $\mathbf{1}$ & $\mathbf{2}$ & $\mathbf{3}$ & $\mathbf{4}$ \\
\hline C0 & $\mathbf{0}$ & 0 & 0 & 10 & 10 & 10 & 10 \\
C1 & 1.5 & 53.0 & 2.71 & 10 & 10 & 10 & 10 \\
C2 & 3 & 50.05 & 5.43 & 10 & 10 & 10 & 10 \\
C3 & $\mathbf{6}$ & 44.13 & 10.86 & 10 & 10 & 10 & 10 \\
C4 & 12 & 32.32 & 21.71 & 10 & 10 & 10 & 10 \\
C5 & 24 & 8.70 & 43.42 & 10 & 10 & 10 & 10 \\
\hline
\end{tabular}

Fuente. Elaboración propia

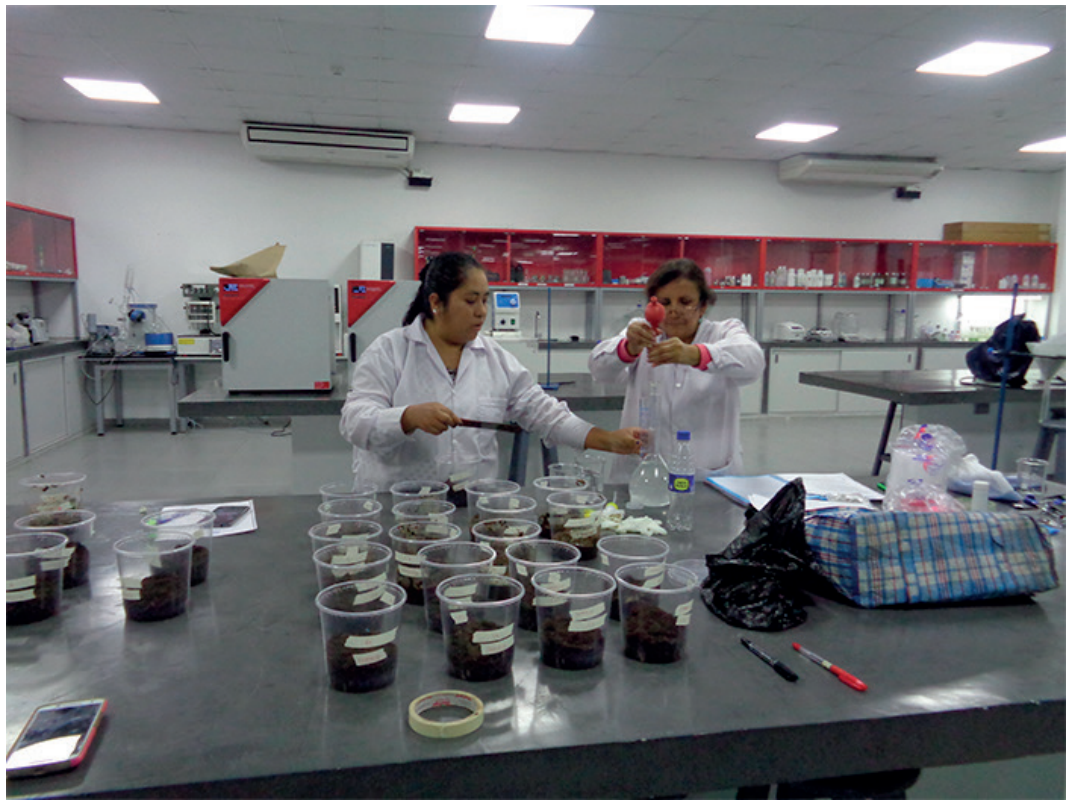

Figura 4. Preparación de soluciones y unidades de muestreo

Vol. KCl: 2.88 g / 132.65 g/L x 1000 ml/L= $21.71 ~ \mathbf{~ m l ~}$ $21.71 \times 1,0883 \mathrm{~g} / \mathrm{ml}=23.63 \mathrm{~g}$

Vol. $\mathbf{H}_{2} \mathbf{O}$ : $(55.95 \mathrm{~g}-23.63 \mathrm{~g}) \times 1 \mathrm{~g} / \mathrm{ml}=\mathbf{3 2 . 3 2} \mathbf{~ m l}$

e. Determinación de los volúmenes de agua y $\mathrm{KCl}$ para una concentración de $24 \mathrm{mg} \mathrm{KCl} / \mathrm{g}$ suelo

Peso KCl: $(240$ g x 24 mg/g x 1 g) / 1000 mg = 5.76 mg

Vol. KCl: 5.76 g / 132.65 g/L x 1000 ml/L= $43.42 ~ \mathbf{~ m l ~}$

$43.42 \times 1,0883 \mathrm{~g} / \mathrm{ml}=47.25 \mathrm{~g}$

Vol. $\mathbf{H}_{2} \mathbf{O}:(55.95 \mathrm{~g}-47.25 \mathrm{~g})$ x $1 \mathrm{~g} / \mathrm{ml}=\mathbf{8 . 7 0} \mathbf{~ m l}$

\subsubsection{Aplicación de las soluciones}

Una vez calculadas las cinco concentraciones a utilizar, con una pipeta de $10 \mathrm{ml}$ se agrega las diferentes concentraciones de $\mathrm{KCl}$ sobre cada una de las unidades experimentales exceptuando los controles y luego la cantidad de agua calculada para todas las unidades. Se homogeniza la preparación y se ingresan en grupos de a 10 las lombrices dentro de las unidades (Guinea León \& Torres Guerrero, 2018).

\section{RESULTADOS}

\subsection{Pruebas ecotoxicológicas}

En el proceso de la prueba de toxicidad de acuerdo con los criterios de aceptabilidad (Ver tabla 6), la mortalidad de las lombrices rojas se registró en relación al grado de concentración, determinándose la $\mathrm{CL}_{50}$ en la concentración $\mathrm{C}_{3} \mathrm{Al}$ cuarto día del experimento (Ver tabla 7) se encontró a los organismos sobrevivientes en la parte superior del sustrato que contiene el recipiente de plástico, cuyos cuerpos presentaban cierta deformación y decoloración a partir de la concentración 2; así como inflamaciones (síndrome de Gozzo o protéico) con textura de color oscuro y blanquecino y abultamiento anormal en la zona clitelar. Asimismo, se ha observado que en los ensayos de las concentraciones 4 y 5 , los movimientos de las lombrices son lentos con dificultad para estirarse y encogerse. 
Tabla 6. Factores de admisión para prueba de toxicidad subcrónica

\begin{tabular}{ll}
\hline Organismo de prueba & Eisenia foetida (lombriz roja) \\
Tipo de prueba & Estática \\
Duración del bioensayo & 14 días \\
Revisiones & $4,7,10$ y 14 días \\
Sustancia de solución & $\mathrm{KCl}$ \\
Agua de dilución & Agua potable \\
Control negativo & Suelo artificial \\
Cantidad requerida de suelo & $7200 \mathrm{~g}$ \\
Cantidad requerida por réplica & $300 \mathrm{~g}$ \\
Humedad del suelo artificial & $65 \%$ \\
pH del suelo artificial & $5-9$ \\
Temperatura & $20 \pm 2{ }^{\circ} \mathrm{C}$ \\
Fotoperiodo & $16: 8$ \\
Edad del organismo de prueba & Estadío adulto (con clitelio) con peso de \\
\hline No de organismos por muestra & 10 \\
№ de réplicas por concentración & 4 \\
Alimento & No \\
Concentraciones de prueba & 5 \\
Punto final & Mortalidad \\
Criterio de aceptabilidad de prueba & $90 \%$ o más sobrevivientes en el control \\
\hline F & \\
\hline
\end{tabular}

Fuente. Elaboración propia

Durante la verificación se detectó la presencia de pequeños organismos como mosquitos y ácaros, como parte del proceso de descomposición de las lombrices muertas, sobre cuyos cuerpos seccionados y derretidos se halló una especie de moho color blanco.

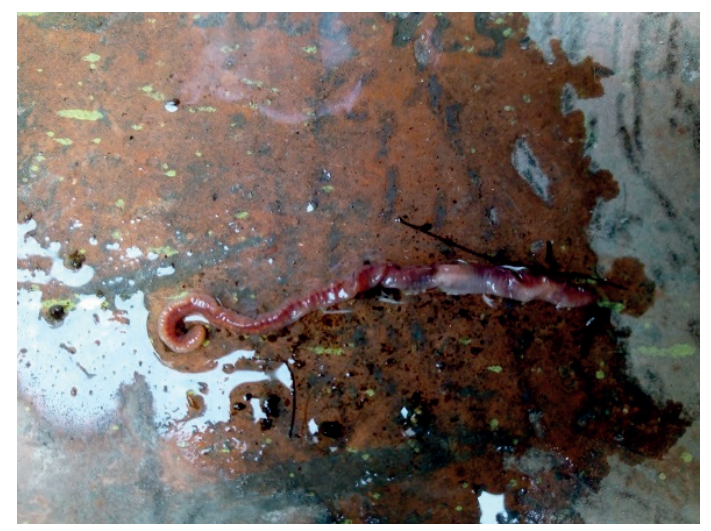

Figura 5. Resultado de pruebas ecotoxicológicas

Tabla 7. $N^{\circ}$ de lombrices rojas sobrevivientes al cuarto día del bioensayo

\begin{tabular}{cccccc}
\hline \multirow{2}{*}{ Concentración } & \multirow{2}{*}{$\mathrm{KCl}(\mathbf{m g} / \mathrm{g})$} & \multicolumn{5}{c}{ Repeticiones } \\
\cline { 2 - 6 } & $\mathbf{1}$ & $\mathbf{2}$ & $\mathbf{3}$ & $\mathbf{4}$ \\
\hline C0 & 0 & 10 & 10 & 10 & 10 \\
$\mathrm{C} 1$ & 1.5 & 9 & 9 & 9 & 10 \\
C2 & 3 & 7 & 8 & 8 & 9 \\
C3 & 6 & 7 & 3 & 5 & 7 \\
C4 & 12 & 4 & 2 & 6 & 1 \\
C5 & 24 & 0 & 1 & 1 & 1
\end{tabular}

Fuente. Elaboración propia
Durante la realización del conteo de los organismos sobrevivientes al día 7 (Ver tabla 8), se obtuvo un total de 85 lombrices vivas de las 200 expuestas a las diferentes concentraciones del cloruro de potasio, representando un $57.5 \%$ de mortandad, así como al día 10 se obtuvo un total de 72 lombrices vivas (Ver tabla 9). Al día 14 del ensayo (Ver tabla 10) se observó un total de 59 lombrices vivas de las 200 expuestas, representando un $70.5 \%$ de mortandad. Al momento de efectuar las revisiones de los organismos vivos, éstos permanecían enterrados en la parte superficial del sustrato de manera agrupada, observándose pérdida de peso y decoloración en la zona del vientre, al mismo tiempo que la textura anillada de sus cuerpos iba desapareciendo, volviéndose flácida y pegajosa.

Tabla 8. № de lombrices rojas sobrevivientes al sétimo día del bioensayo

\begin{tabular}{cccccc}
\hline \multirow{2}{*}{ Concentración } & \multirow{2}{*}{$\mathrm{KCl}(\mathrm{mg} / \mathrm{g})$} & \multicolumn{5}{c}{ Repeticiones } \\
\cline { 3 - 6 } & $\mathbf{1}$ & $\mathbf{2}$ & 3 & $\mathbf{3}$ \\
\hline C0 & 0 & 10 & 10 & 10 & 10 \\
C1 & 1.5 & 9 & 8 & 7 & 10 \\
C2 & 3 & 4 & 8 & 8 & 8 \\
C3 & 6 & 7 & 3 & 5 & 3 \\
C4 & 12 & 1 & 2 & 2 & 0 \\
C5 & 24 & 0 & 0 & 0 & 0 \\
\hline
\end{tabular}

Fuente. Elaboración propia

Tabla 9. № de lombrices rojas sobrevivientes al décimo día del bioensayo

\begin{tabular}{cccccc}
\hline \multirow{2}{*}{ Concentración } & \multirow{2}{*}{$\mathrm{KCl}(\mathrm{mg} / \mathrm{g})$} & \multicolumn{5}{c}{ Repeticiones } \\
\cline { 3 - 6 } & $\mathbf{1}$ & $\mathbf{2}$ & $\mathbf{3}$ & $\mathbf{4}$ \\
\hline C0 & 0 & 10 & 10 & 10 & 10 \\
$\mathrm{C} 1$ & 1.5 & 9 & 8 & 7 & 10 \\
C2 & 3 & 4 & 8 & 8 & 7 \\
C3 & 6 & 6 & 1 & 3 & 1 \\
C4 & 12 & 0 & 0 & 0 & 0 \\
C5 & 24 & 0 & 0 & 0 & 0 \\
\hline
\end{tabular}

Fuente. Elaboración propia

Tabla 10. $\mathrm{N}^{0}$ de lombrices rojas sobrevivientes al catorceavo día del bioensayo

\begin{tabular}{cccccc}
\hline \multirow{2}{*}{ Concentración } & \multirow{2}{*}{$\mathrm{KCl}(\mathrm{mg} / \mathrm{g})$} & \multicolumn{5}{c}{ Repeticiones } \\
\cline { 2 - 6 } & $\mathbf{1}$ & $\mathbf{2}$ & $\mathbf{3}$ & $\mathbf{4}$ \\
\hline C0 & $\mathbf{0}$ & 10 & 10 & 10 & 10 \\
$\mathrm{C} 1$ & 1.5 & 7 & 8 & 5 & 8 \\
C2 & 3 & 2 & 7 & 7 & 7 \\
C3 & 6 & 4 & 3 & 2 & 1 \\
C4 & 12 & 0 & 0 & 0 & 0 \\
C5 & 24 & 0 & 0 & 0 & 0 \\
\hline
\end{tabular}

Fuente. Elaboración propia

\subsection{Pruebas estadísticas}

El análisis de probabilidad y estadística de los resultados obtenidos fue realizado a través de los programas Probit 1.5 y TSK, con límites de confianza del $95 \%$ (Ver figura 6 - 8). 


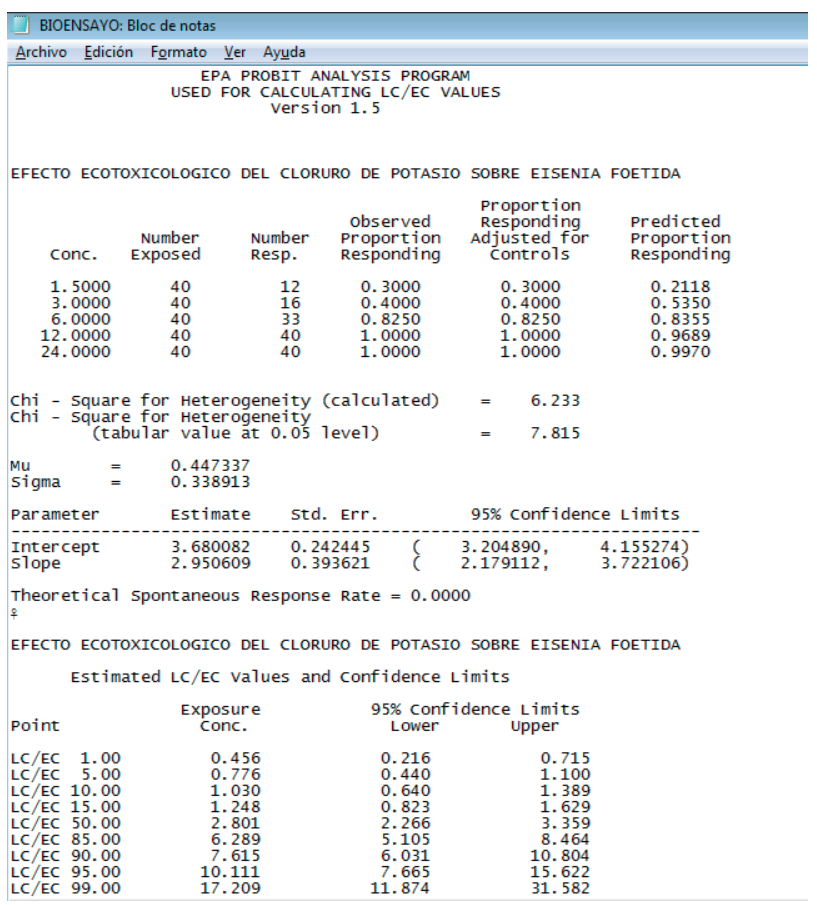

Figura 6. Análisis y estimación de valores Probit 1.5

\section{DISCUSIÓN}

De acuerdo resultados se observa que la mortandad, se ha ido incrementando conforme pasaban los días, en las concentraciones $\mathrm{C}_{2}$ y $\mathrm{C}_{3}$, asimismo para los ensayos sin ninguna concentración de cloruro de potasio, se observa que las 10 especies sometidas, ha sobrevivido, hasta los 14 días, por lo que se considera que el suelo al cual fueron colocadas tenía una buena calidad, para su supervivencia.

Para las especies sometidas a la concentración $\mathrm{C}_{5}$, no se encontró ninguna especie viva, y más por el contrario, se encontraron especies descompuestas y seccionadas, entendiéndose que esta concentración de sales es perjudicial al $100 \%$ de las especies sometidas.

Respecto a la concentración $\mathrm{C}_{4}$, si bien en un conteo de 7 días, existían aun especies vivas, para el conteo del día 14 , no se encontró ninguna especie viva.

\section{CONCLUSIONES}

De acuerdo con el análisis estadístico efectuado en el programa Probit versión 1.5, la $\mathrm{CL}_{50}$ estimada para el presente ensayo a los 14 días de exposición fue de 2.801 ,

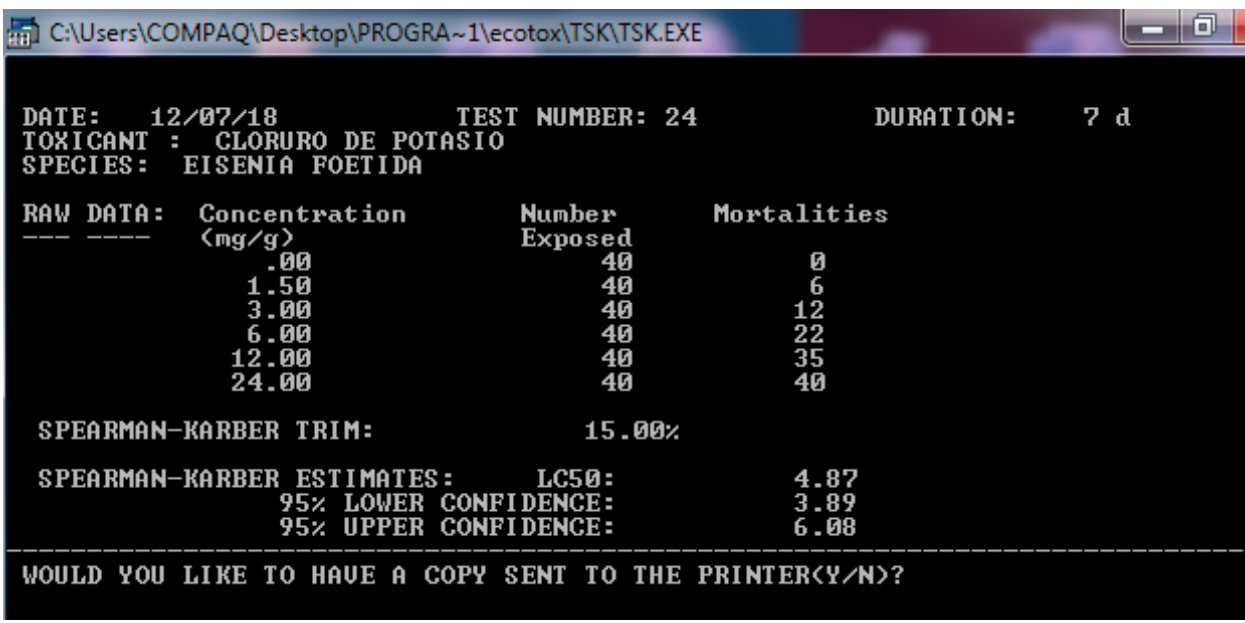

Figura 7. Análisis y estimación de valores TSK al día 7 del bioensayo

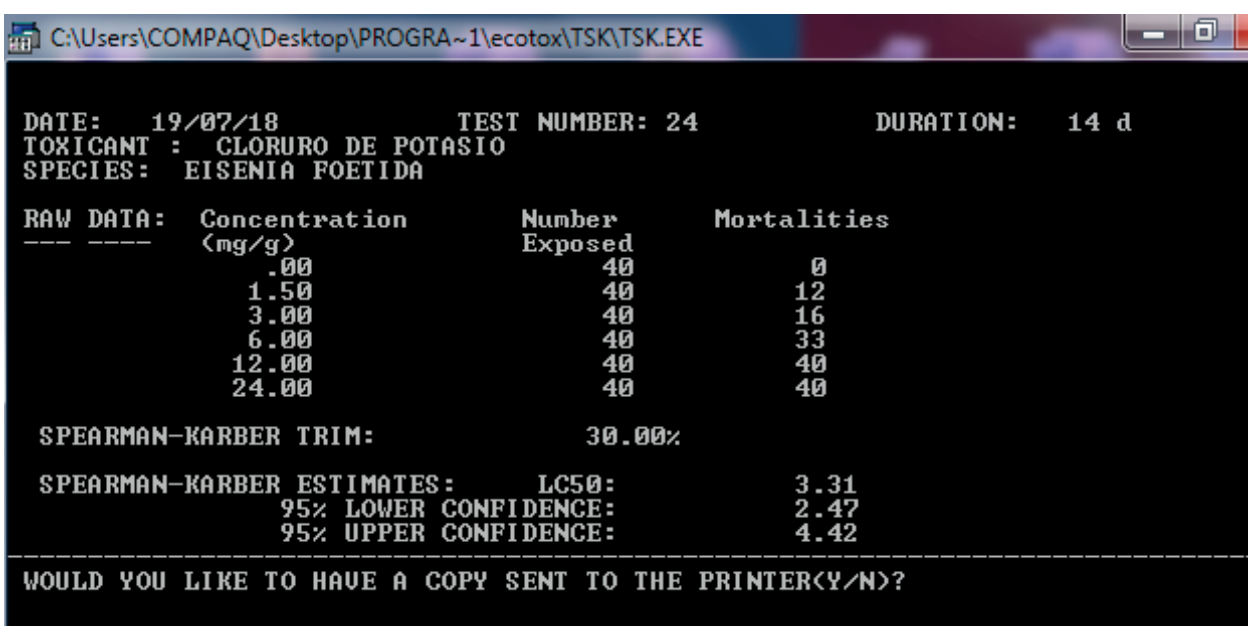

Figura 8. Análisis y estimación de valores TSK al día 14 del bioensayo 
siendo sus límites de confianza de 2.266 y 3.359; asimismo, según el análisis estadístico efectuado en el programa TSK versión 1.5 , la $\mathrm{CL}_{50}$ estimada para los ensayos a los 7 y 14 días fue de 4.87 y 3.31 , respectivamente.

A mayor concentración de la solución con cloruro de potasio, existirá mayor mortandad de organismos, siendo preciso mencionar que el peso puede ser una condición influyente en dicha mortandad, ya que al momento de efectuar el conteo final de las lombrices se ha observado que las sobrevivientes son las de mayor tamaño. El cloruro de potasio es un tóxico para el organismo de prueba, no sólo a nivel metabólico, sino que también a nivel físico, ya que a mayor concentración se ha destruido el cuerpo de los organismos.

Los valores de $\mathrm{pH}$ del suelo artificial se han incrementado a medida que han transcurrido los días de exposición, debido a que la descomposición de los organismos muertos ha influenciado en la acidificación del sustrato. Finalmente, se puede concluir que los resultados de esta prueba con Eisenia foetida son confiables, debido a que los ensayos del grupo control conserva el $100 \%$ de organismos sobrevivientes.

\section{AGRADECIMIENTOS}

Expresamos un sincero agradecimiento a nuestro docente de Ecotoxicología Acuática, biólogo Christian Paredes Espinal, quien fue nuestro guía y motivación para realizar la presente investigación.

\section{REFERENCIAS}

Carlosama Camacho, J., \& Cepeda Rojas, C. A. (2018). Determinación de la concentración letal 50 (cl50) en la lombriz californiana, eisenia foetida (lombricidae) de dos fungicidas de uso agrícola con el mismo principio activo (difenoconazol) [Universidad Santo Tomás. Bogotá, Colombia]. http://repository.usta.edu.co/handle/11634/2478

Chang Marrero O., Jiménez Aguila E., Hernández Castañedo Z., Armengol Hernández R., Meneses-Marcel A., H. de M. Y. M. Y. (2018). Evaluación de la toxicidad del Vitrofural en agar sobre lombriz de tierra. Biotecnología Vegetal, 18(1), 31-36. https://revista.ibp.co.cu/index.php/BV/article/view/573/html
Cuevas, C., \& Cerrato, R. F. (2002). Ensayo de toxicidad subcrónica con la lombriz de tierra Eisenia andrei. 275284. http://www2.inecc.gob.mx/publicaciones2/libros/573/ cap14.pdf

Gomez Hernandez, A. (2014). Evaluacion de la toxicidad de suelos mediante un bio-ensayo con la lombriz de tierra Eisenia fetida [Universidad Nacional de Colombia]. https://repositorio.unal.edu.co/bitstream/handle/ unal $/ 48558 / 822152.2014$.pdf? sequence $=1$

Guinea León, C. D., \& Torres Guerrero, I. J. (2018). Determinación de la concentración letal 50 (cl50) en la lombriz californiana, eisenia foetida (lombricidae) de dos fungicidas de uso agrícola con el mismo principio activo (difenoconazol). https://repository.usta.edu.co/ handle/11634/10664?show=full

Iannacone Oliver, J. A. (2017). Toxicidad de sales de amonio sobre la lombriz de tierra (Eisenia foetida). Facultad de Ciencias Biologicas-Universidad Ricardo Palma, 28. http://repositorio.urp.edu.pe/bitstream/handle/ URP/1559/2017InformeFinal\%C2\%A0Eisenia\%2022\%20 feb2018.pdf?sequence=1

Mancebo Rodríguez, A., Estrada Ortiz, J., González Triana, C., González Torres, Y., González Navarro, B., \& Bada Barro, A. M. (2011). Evaluación ecotoxicológica de dos derivados del nim en lombriz de tierra y abejas. Revista de Toxicologia, 28(2), 147-151. https://dialnet.unirioja.es/ servlet/articulo? codigo $=4032141$

Peña Córdova, K. D. (2018). Evaluación de riesgo ambiental de los pesticidas metamidofos, alfa-cipermetrina y su mezcla en Eisenia andrei. http://repositorio.lamolina.edu.pe/ bitstream/handle/UNALM/3529/peña-cordova-katherindeira.pdf? sequence $=5 \&$ is Allowed $=\mathrm{y}$

Rubiano Rodríguez, V. E. (2019). Informe técnico evaluación ecotoxicológica de plaguicidas sobre eisenia foetida (p. 46). https://expeditiorepositorio.utadeo.edu.co/ bitstream/handle/20.500.12010/7910/Trabajo de grado. pdf? sequence $=1 \&$ is Allowed $=y$ 